\title{
STUDIES ON GRAFT COPOLYMERIZATION OF 2-HYDROXYETHYLMETHACRYLATE ONTO KAPPA- CARRAGEENAN INITIATED BY CERIC AMMONIUM NITRATE
}

\author{
MOHAMMAD SADEGHI ${ }^{* 1}$ AND HOSSEIN HOSSEINZADEH
}

\author{
${ }^{1}$ Department of Chemistry, Science Faculty, Islamic Azad University, Arak Branch, Arak, Iran. Tel: +98-861-3670017, Fax: +98-861-3670017 \\ ${ }^{2}$ Department of Chemistry, University of Payame Noor, West Azerbaijan, Miandoab, Iran. Tel: +98-481-2227777, Fax: +98-481-2227997
}

(Received: June 17, 2010 - Accepted: November 5, 2010)

\begin{abstract}
The polysaccharide, kappa-carrageenan $(\mathrm{kC})$, has been chemically modified by graft copolymerization of 2-hydroxyethylmethacrylate (HEMA) in an aqueous medium using ceric ammonium nitrate (CAN) as an initiator under argon atmosphere. A plausible reaction mechanism of grafting has been suggested. Evidence of grafting was obtained by comparison of FTIR spectra of $\mathrm{kC}$ and homopolymer-free $\mathrm{kC}$ - $g$-poly(HEMA) as well as solubility characteristics and gravimetric analysis of the products. The optimum reaction conditions affording maximum grafting ratio and add-on value have been determined. The grafting parameters have been found to increase with increasing in $\mathrm{kC}, \mathrm{CAN}$, and HEMA concentrations as well as polymerization time and temperature, up to a certain value, but these parameters decrease on further increasing in reaction conditions.
\end{abstract}

Keywords: polymer synthesis, carrageenan, 2-hydroxyethylmethacrylate, graft copolymerization, ceric ammonium nitrate

\section{INTRODUCTION}

Graft copolymerization of hydrophilic and hydrophobic vinyl monomers is a well-known technique employed by polymer chemists for significantly modifying the chemical and physical properties of the synthetic or natural starting materials with minimum degradation of the original properties ${ }^{1-7}$. Graft copolymers are prepared by first generating free radicals on the polysaccharide backbone and then allowing these radicals to serve as macroinitiators for the vinyl monomers. These biodegradable and low cost graft copolymers, with new properties can be used in many applications such as textiles, paper industry, agriculture, medical treatment, in petroleum industry as flocculants and thickening agents ${ }^{2,3}$ and also development of selective permeable membranes, ${ }^{8}$ sorption agents, ${ }^{9}$ and in fabrication of drug delivery systems. ${ }^{10,11}$

Grafting can be performed using free radical initiators, redox systems or photochemical process. Cerium in its tetravalent state $\left(\mathrm{Ce}^{4+}\right)$ is a versatile oxidizing agent that through various redox reactions with many different organic substrates can create free radicals capable of initiating vinyl polymerizations ${ }^{1}$. Since the discovery of ceric ammonium nitrate (CAN) as an initiatir by Mino and Kizerman ${ }^{12}$, it has been widely used by many investigators for initiating graft copolymerization of vinyl monomers onto various natural and synthetic polymers. For example, graft copolymerization of acrylamide ${ }^{13}$, acrylonitrile ${ }^{14}$, and methacrylonitrile ${ }^{15}$ were performed using Ce (IV) as an initiator.

Though much work has been reported on the grafting of 2-hydroxyethylmethacrylate (HEMA) onto various polysaccharides, but a literature survey reveals that no paper has been reported in the case of HEMA grafting onto kappa-carrageenan $(\mathrm{kC})$. Therefore, The present investigation deals with the detailed study of some major factors which affect graft copolymerization of HEMA onto kappa-carrageenan, initiated by CAN in aqueous medium with a view to elucidate the grafting mechanism.

The chosen polysaccharide for modification, i.e. kappa-carrageenan, $\mathrm{kC}$, is the most well-known and most important type of carrageenan family. Carrageenan is a collective term for linear sulfated polysaccharides that are obtained commercially by alkaline extraction of certain species of red seaweeds ${ }^{16}$. Schematic diagram of the idealized structure of the repeat units for the $\mathrm{kC}$, is framed in Scheme 1.

Carrageenan is not a single biopolymer but a mixture of water-soluble, linear, sulfated galactans. They are composed of alternating 3-linked $\beta$-Dgalactopyranose (G-units) and 4-inked $\beta$-D-galactopyranose (D-units) or 4-linked 3,6-anhydrogalactose (DA-units) forming the "ideal" disacchariderepeating unit of carrageenans. The sulfated galactans are classified according to the presence of the 3,6-anhydrogalactopyranose on the 4-linked residue and the position and number of sulfate groups. The corresponding IUPAC name and letter code for kappa-carrageenan are carrageenose 4'-sulfate and G4S-DA.

\section{MATERIALS AND METHODS}

\section{Material}

The polysaccharide, kappa-carrageenan $(\mathrm{kC}, \mathrm{MW}=100,000$, from Condinson Co., Denmark) was of analytical grade and was used as received. Ceric ammonium nitrate (CAN) was purchased from Merck and was used without purification. It was as freshly prepared $0.1 \mathrm{M}$ solution in $1 \mathrm{~N}^{-\mathrm{NO}_{3}}$. The monomer, 2-hydroxyethyl methacrylate (HEMA, from Merck) was used after distillation for removing inhibitor.

\section{Grafting procedure}

Graft copolymerization of 2-hydroxyethylmethacrylate onto $\mathrm{kC}$ was carried out with CAN radical initiator under argon atmosphere. In a $100 \mathrm{~mL}$ flask, certain amount of $\mathrm{kC}(0.5-3.0 \mathrm{~g})$ was dissolved in $50 \mathrm{~mL}$ of degassed distilled water. The flask was placed in a water bath with desired temperature $\left(40-100{ }^{\circ} \mathrm{C}\right)$. A given amount of monomer, HEMA (1.0-5.0 g), was added to the flask and the mixture was stirred for $10 \mathrm{~min}$. Then the initiator solution (0.5-6.0 mL of $0.1 \mathrm{~mol} / \mathrm{L}$ acidic solution of CAN) was added to the mixture and continuously stirred for certain times (30-180 min). An inert gas (argon) was gently bubbled into the reactor to remove the oxygen during the graft copolymerization reaction. The product was then worked up with methanol $(200 \mathrm{~mL})$ and dried in oven at $50^{\circ} \mathrm{C}$ for $5 \mathrm{~h}$.

\section{Homopolymer extraction}

The graft copolymer, $\mathrm{kC}$ - $g$-poly(HEMA), was freed from poly (HEMA) homopolymer, by pouring $0.50 \mathrm{~g}$ of the product in $50 \mathrm{~mL}$ of dimethyl formamide solution. The mixture was stirred gently at room temperature for $24 \mathrm{~h}$. After complete removal of the homopolymer by filtration of the $\mathrm{kC}-\mathrm{g}$ poly(HEMA) copolymer, the product was washed with methanol and dried in oven at $50^{\circ} \mathrm{C}$ to reach a constant weight.

\section{Infrared analysis}

The samples were crushed with $\mathrm{KBr}$ to make pellets. Spectra were taken on an ABB Bomem MB-100 FTIR spectrophotometer.

\section{Thermal analysis}

Thermogravimetric analyses were performed on a Universal V4.1D TA Instruments (SDT Q600) with 8-10 mg samples on a platinum pan under nitrogen atmosphere. Experiments were performed at a heating rate of $10{ }^{\circ} \mathrm{C} /$ min until $600^{\circ} \mathrm{C}$.

\section{Grafting parameters}

The grafting parameters, i.e. grafting rati (Gr\%), add-on value ( $\mathrm{Ad} \%)$, and 
homopolymer content $(\mathrm{Hp} \%)$, used to characterize the nature of the copolymer are defined and calculated using the following equations ${ }^{1,2}$ :

$$
\begin{aligned}
& \mathrm{Gr} \%=100\left(\mathrm{~W}_{2}-\mathrm{W}_{0}\right) / \mathrm{W}_{0} \\
& \mathrm{Ad} \%=100\left(\mathrm{~W}_{2}-\mathrm{W}_{0}\right) / \mathrm{W}_{2} \\
& \mathrm{Hp} \%=100\left(\mathrm{~W}_{1}-\mathrm{W}_{2}\right) / \mathrm{W}_{1}
\end{aligned}
$$

where $\mathrm{W}_{0}, \mathrm{~W}_{1}$, and $\mathrm{W}_{2}$ are the weight of the initial substrate, total product (copolymer and homopolymer), and pure graft copolymer (after DMF extraction), respectively.

\section{RESULTS AND DISCUSSION}

\section{Grafting mechanism}

A general reaction mechanism for HEMA grafting onto $\mathrm{kC}$ backbones is shown in Scheme 1. At the first step, a complex between the $\mathrm{Ce}^{4+}$ ion with the oxygen atom at the $\mathrm{C}-3$ position and the hydroxyl group at the $\mathrm{C}-2$ position was formed. This ceric- $\mathrm{kC}$ complex is then dissociated to produce $\mathrm{kC}$ macroradicals. The monomer molecules, which are in vicinity of the macroradical sites, become acceptor of $\mathrm{kC}$ radicals resulting in chain initiation and thereafter themselves become free radical donor to the neighboring molecules leading to propagation. These grafted chains are terminated by coupling to give the graft copolymer.

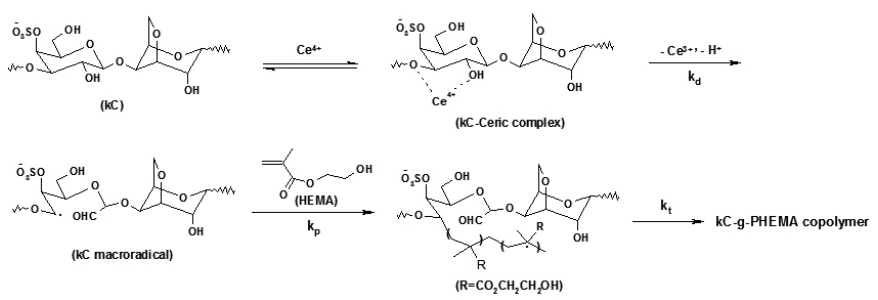

Scheme 1. A brief proposed mechanism for ceric-induced grafting of poly(2-hydroxyethylmethacrylate ) onto kappa-carrageenan.

\section{Evidence for grafting}

\section{FTIR analysis}

Structural changes of $\mathrm{kC}$ and its graft copolymer were confirmed by FTIR spectroscopy. The FTIR spectrum of $\mathrm{kC}$ and the final grafted copolymer, $\mathrm{kC}$ $g$-poly(HEMA), was shown in Figure 1. The IR spectrum of $\mathrm{kC}$ shows peaks at $840,914,1019$, and $1225 \mathrm{~cm}^{-1}$ could be related to $\beta$-D-galactopyranose-4sulfate, 3,6-anhydro- $\beta$-D-galactopyranose, glycosidic linkage, and ester sulfate stretching of kC, respectively (Figure 1a). The broad band at $3200-3400 \mathrm{~cm}^{-1}$ is due to stretching of $-\mathrm{OH}$ groups of $\mathrm{kC}$. In the spectrum of homopolymer-free $\mathrm{kC}$-g-poly(HEMA), the strong peak at $1733 \mathrm{~cm}^{-1}$ could be assigned to the $\mathrm{C}=\mathrm{O}$ stretching in the ester group from the poly(HEA) grafted onto $\mathrm{kC}$ backbones.

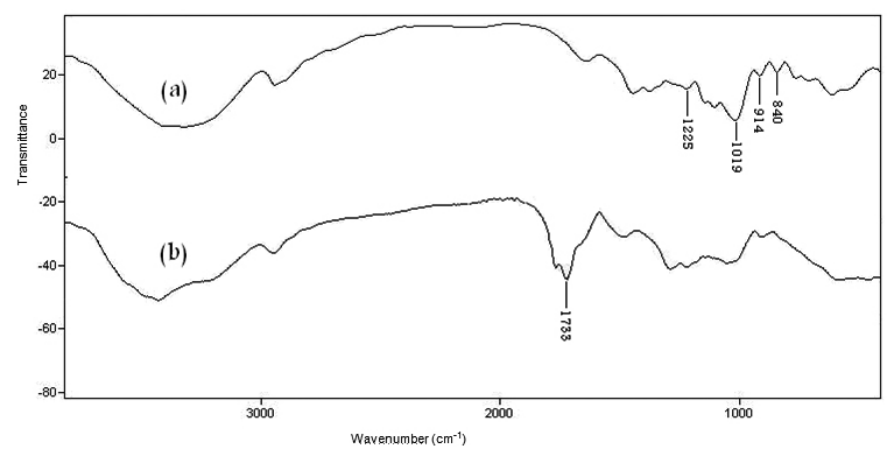

Figure 1. FTIR spectra of (a) $\mathrm{kC}$ and (b) graft copolymer, kC-g-PHEMA.

\section{Thermogravimetric behavior}

The grafting was also supported by thermogravimetric analysis (Fig. 2). TGA of kappa-carrageenan (Fig. 2a) shows a weight loss in two distinct stages. The first stage ranges between 15 and $120{ }^{\circ} \mathrm{C}$ and shows about $17 \%$ loss in weight. This may correspond to the loss of adsorbed and bound water. ${ }^{17}$ No such inflexion was observed in the TGA curve of kC- $g$-PHEMA. This indicated that the grafted copolymers were resistant to moisture absorption. The second stage of weight loss starts at $3300^{\circ} \mathrm{C}$ and continues up to $440{ }^{\circ} \mathrm{C}$ during which there was $60 \%$ weight loss due to the degradation of kappa-carrageenan. Grafted samples, however, show almost different behavior of weight loss between 15 and $550{ }^{\circ} \mathrm{C}$ (Fig. 2b). The first stage of weight loss starts at $205^{\circ} \mathrm{C}$ and continues up to $330{ }^{\circ} \mathrm{C}$ due to the degradation of kappa-carrageenan. The second stage from 370 to $480{ }^{\circ} \mathrm{C}$ may contribute to the decomposition of different structure of the graft copolymer. The appearance of these stages indicates the structure of kappa-carrageenan chains has been changed, which might be due to the grafting of PHEMA chains. In general, the copolymer had lower weight loss than kappa-carrageenan. This means that the grafting of kappa-carrageenan increases the thermal stability of kappa-carrageenan in some extent.

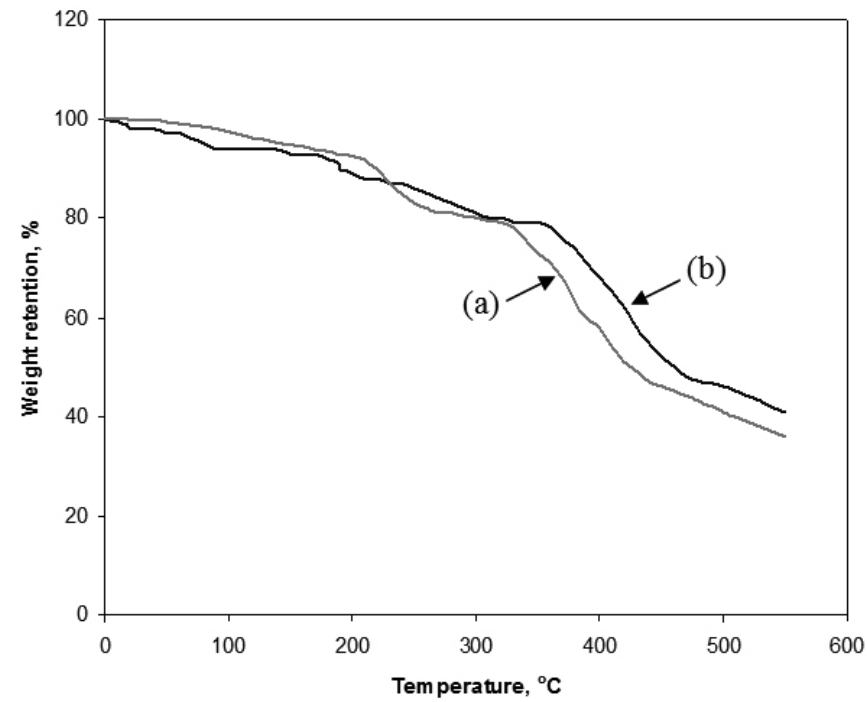

Figure 2. TGA curves of (a) kC and (b) kC-g-PHEMA.

\section{Solubility test}

To obtain an additional evidence of grafting, solubility difference between the grafted and the non-grafted polymer was used. $\mathrm{kC}$ and poly(HEMA) are soluble in water and DMF, respectively. When a reaction product was extracted with DMF and alternatively with water for $24 \mathrm{~h}$, an insoluble solid still remained. A physical mixture of $\mathrm{kC}$ and poly(HEMA) was treated in the same way and was found to dissolve completely. Therefore, it is obvious that the resulted graft copolymer was not a simple physical mixture, but some chemical bonds must exist between the $\mathrm{kC}$ substrate and poly(HEMA) macromolecules. In addition to the formation of graft copolymers, crosslinking between the chains of kappa-carrageenan may also take place. This was evident by the reaction between kappa-carrageenan and the initiator, in the absence of monomers, giving a product with reduced solubility (unpublished data).

Moreover, because of insolubility of the graft copolymer and PHEMA in water and in the HEMA monomer, respectively, the polymerization reaction proceeds as a heterogeneous process and the formed copolymer precipitate during the process. This observation practically proves that the grafting reaction was performed.

\section{Gravimetric analysis}

The graft copolymerization reaction was monitored gravimetrically. Increase in the mass of $\mathrm{kC}$, after extraction of homopolymer, was taken as evidence for grafting. This weight gain in $\mathrm{kC}$ forms the basis for the determination of the grafting parameters.

\section{Reaction rate}

The rates of polymerization $\left(\mathrm{R}_{\mathrm{p}}\right)$ and graft copolymerization $\left(\mathrm{R}_{\mathrm{g}}\right)$ may be evaluated as measures of the rate of monomer disappearance by using the following equations ${ }^{18}$ :

The calculation of $R_{p}$ values may be of significant importance in confirming a proposed reaction mechanism and kinetics. Therefore, we investigated the relation between rate of graft copolymerization and concentration of CAN, HEMA and kC. Figures 3-5 show that the plots of Rp versus the monomer concentration, [HEMA], half-order of the initiator concentration, 
$\left[\mathrm{Ce}^{+4}\right]^{1 / 2}$ and the polysaccharide concentration, $[\mathrm{kC}]^{1 / 2}$, are linear. This is in agreement with a modified kinetic scheme already explored for CAN-initiated acrylonitrile grafting onto carboxymethyl cellulose ${ }^{19}$. The statement of rate of polymerization according to the scheme is as follows:

$$
\mathrm{Rp}=\mathrm{k}_{\mathrm{p}}\left(\mathrm{K} \mathrm{k}_{\mathrm{d}} / \mathrm{k}_{\mathrm{t}}\right)^{1 / 2}[\mathrm{kC}]^{1 / 2}\left[\mathrm{Ce}^{+4}\right]^{1 / 2}[\text { HEMA }]
$$

The coefficient $\mathrm{K}$ is the equilibrium constant, $\mathrm{k}, \mathrm{k}_{\mathrm{d}}$, and $\mathrm{k}$ are the rate constants for propagation, $\mathrm{kC}-$ ceric complex dissociation, and termination reactions, respectively. Therefore, we preliminarily conclude that the CANinitiated grafting of HEMA onto $\mathrm{kC}$ is also fitted with this kind of rate statement.

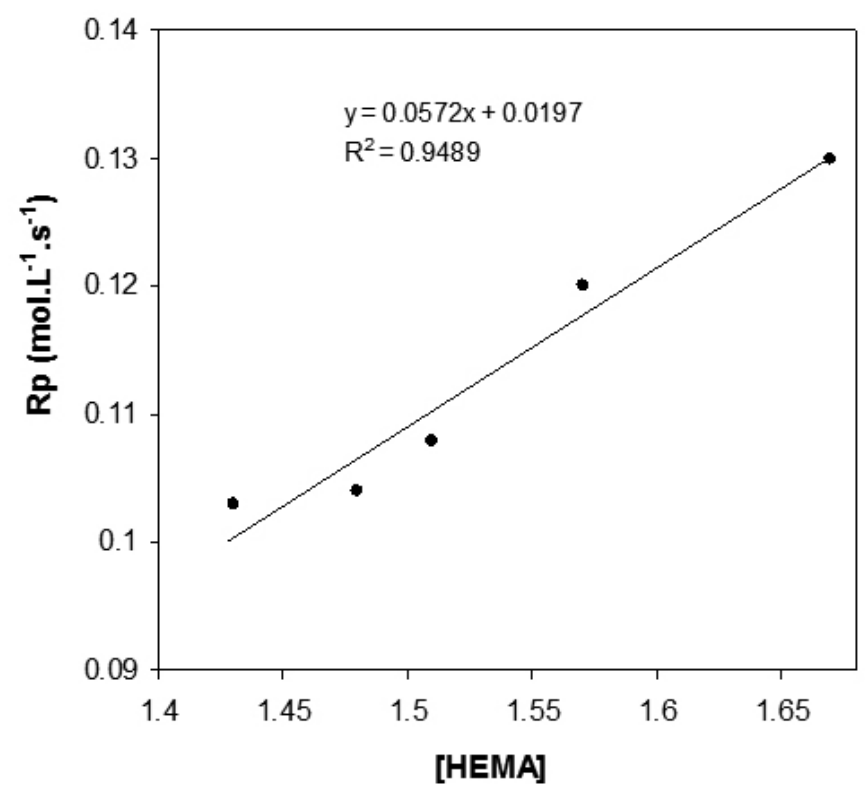

Figure 3. Plot of $\mathrm{Rp}$ versus monomer concentration.

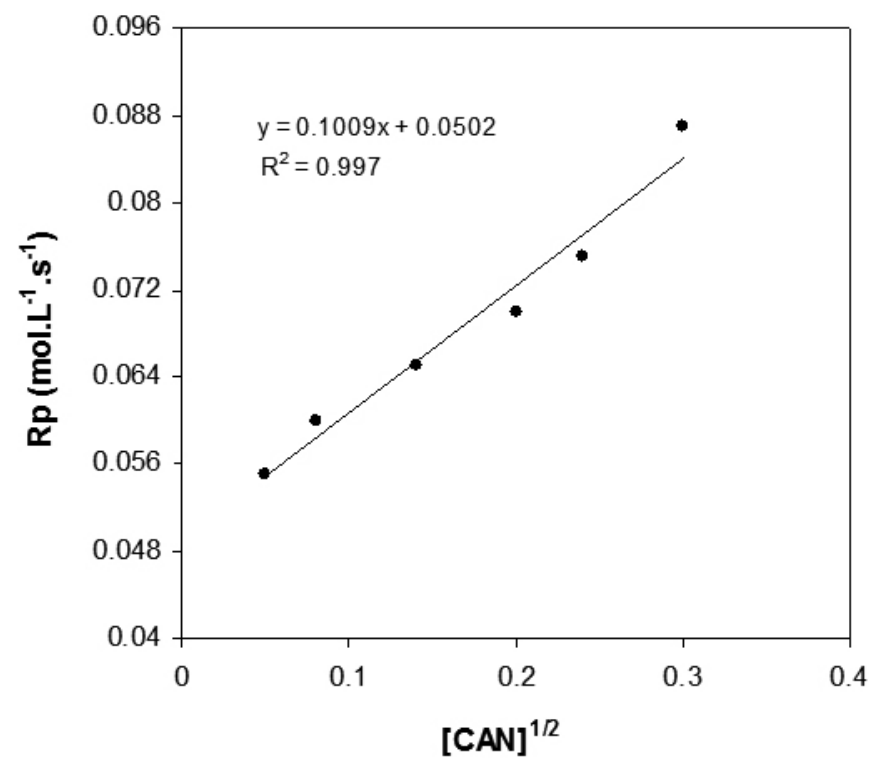

Figure 4. Plot of $\mathrm{Rp}$ versus initiator concentration.

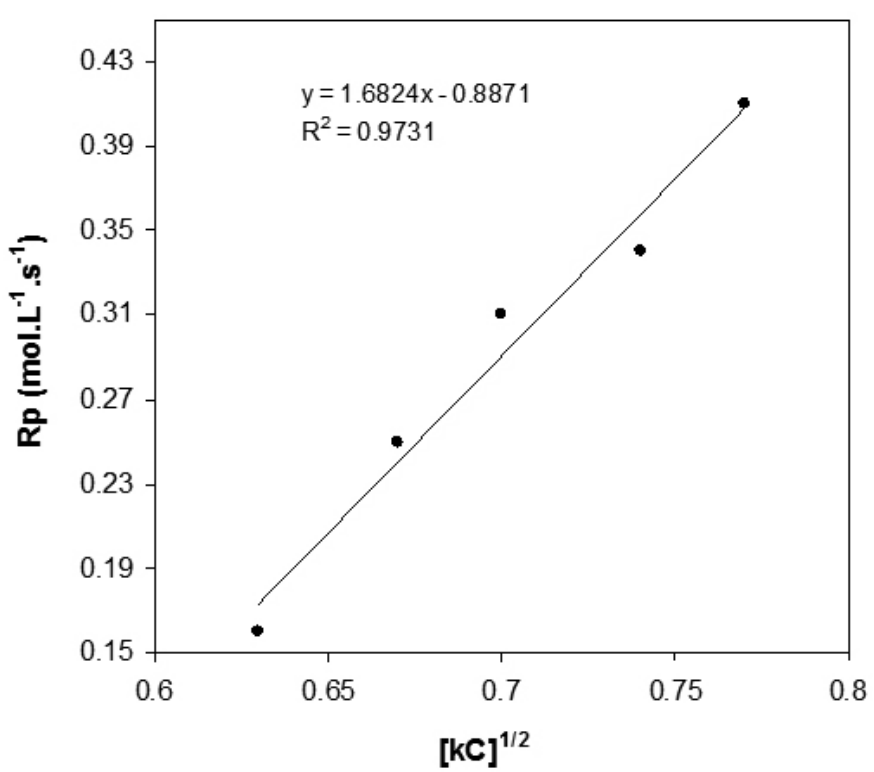

Figure 5. Plot of $\mathrm{Rp}$ versus polysaccharide concentration.

The overall activation energy (Ea) of the graft polymerization reaction was calculated by using of the Equation (5) and the slope of the plot LnRg versus $1 / T$ (Figure 6) based on Arrhenius relationship $\left[\mathrm{k}_{\mathrm{p}}=\mathrm{A} \exp (-\mathrm{Ea} / \mathrm{RT})\right]$. Therefore, Ea for the graft copolymerization was found to be $16.85 \mathrm{~kJ} / \mathrm{mole}$.

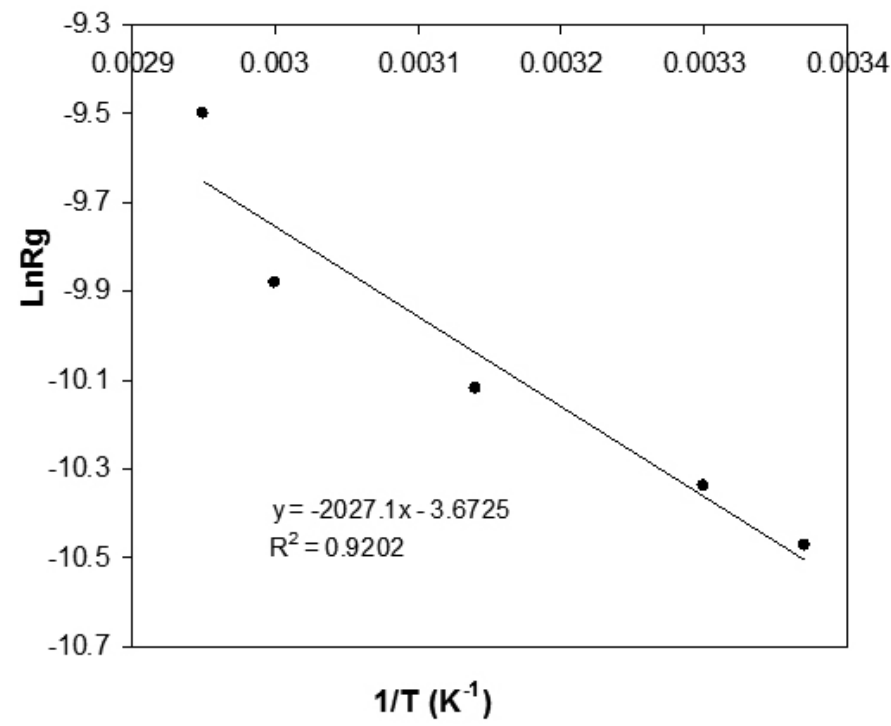

Figure 6. Plot of LnRg versus 1/T for estimating the activation energy of the graft polymerization reaction.

Influence of reaction conditions on grafting parameters

Since the grafting parameters depend upon a large number of variables, the effect of these variables was investigated. During this study, one of the reaction conditions was varied and the other parameters were kept constant.

\section{Effect of initiator concentration}

Grafting of HEMA onto $\mathrm{kC}$ backbones was carried out at various initiator concentrations $(0.001-0.012 \mathrm{~mol} / \mathrm{L})$, as shown in Figure 7. It has been observed that the $\% \mathrm{Gr}$ and $\% \mathrm{Ad}$ increase initially on increasing the CAN concentration up to $0.004 \mathrm{~mol} / \mathrm{L}$, but decrease with further increase in initiator concentration. The initial increase in $\% \mathrm{Gr}$ and $\% \mathrm{Ad}$ may be ascribed to the increase of the active sites on the on the backbone of the $\mathrm{kC}$ arising from the attack of $\mathrm{Ce}^{4+}$. The decrease of grafting parameters at higher concentration of CAN may be due to 
(a) oxidative degradation of $\mathrm{kC}$ chains by excess $\mathrm{Ce}^{4+}$ ions, (b) an increase in the termination reaction of the chain radicals via bimolecular collision because of an increased population of macroradicals produced, and (c) enhancement in homopolymerization reaction. These observations are in agreement with similar observations reported by others ${ }^{20,21}$.

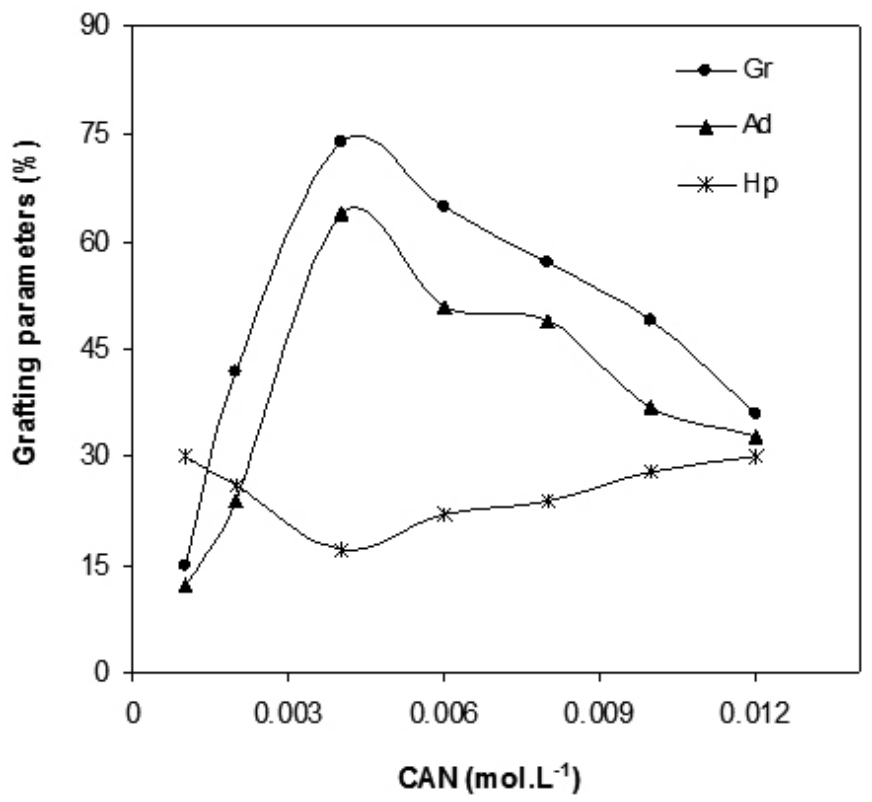

Figure 7. Effect of initiator concentration on the grafting parameters.

Reaction conditions: $\mathrm{kC} 2 \mathrm{wt} \%$, HEMA $0.51 \mathrm{~mol} . \mathrm{L}^{-1}$, temperature $60^{\circ} \mathrm{C}$, time $80 \mathrm{~min}$

\section{Effect of monomer concentration}

The HEMA concentration was varied from 0.17 to $0.85 \mathrm{~mol} / \mathrm{L}$ to study its effects on grafting parameters (Figure 8). These parameters were found to be increased by enhancement of HEMA concentration from 0.17 up to $0.60 \mathrm{~mol} / \mathrm{L}$. This behavior can be attributed to the increase of monomer concentration in the vicinity of the $\mathrm{kC}$ backbone and consequent greater availability and enhancement chances for molecular collisions of the reactants. The decrease in $\% \mathrm{Gr}$ and $\% \mathrm{Ad}$ after a certain level of HEMA $(0.60 \mathrm{~mol} / \mathrm{L})$ is probably due to preferential homopolymerization over graft copolymerization as well as increasing the viscosity of reaction medium, which hinders the movement of free radicals. Needless to say, the increase in the chain transfer to monomer molecules may be other possible reason for the diminished grafting at higher HEMA concentrations. Similar observations have been reported for the grafting of ethyl acrylate onto cellulose ${ }^{22}$ and methyl acrylate onto starch ${ }^{23}$.

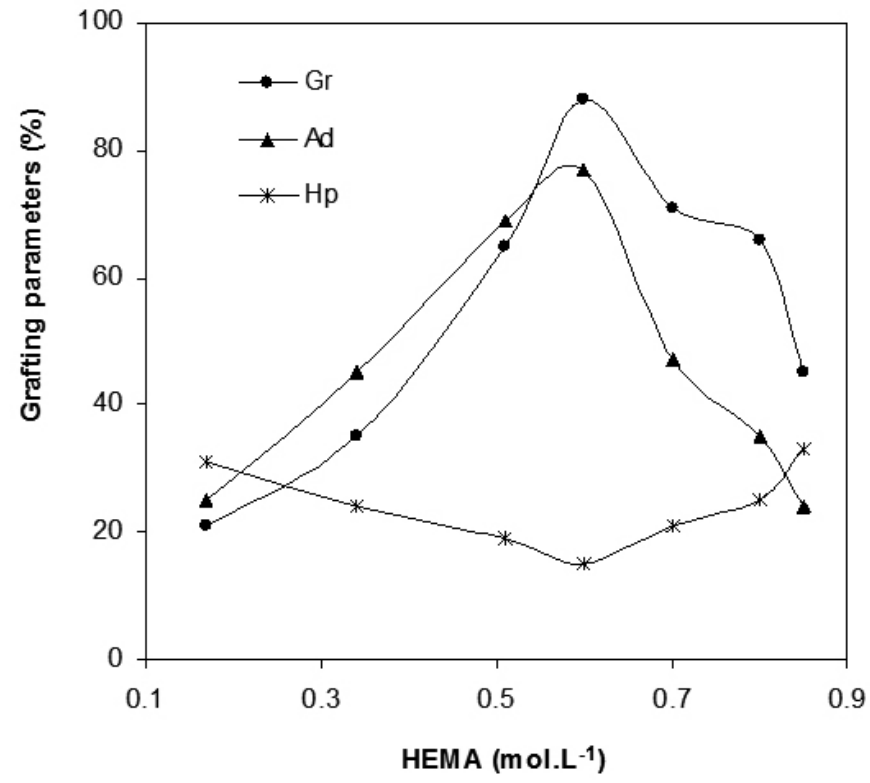

Figure 8. Effect of the monomer concentration on the grafting parameters.

Reaction conditions: $\mathrm{kC} 2 \mathrm{wt} \%$, CAN 0.004 mol. $\mathrm{L}^{-1}$, temperature $60^{\circ} \mathrm{C}$, time $80 \mathrm{~min}$.

\section{Effect of $k C$ concentration}

The results obtained by changing the polysaccharide concentration for the graft polymerization are presented in Figure 9. It is evident from the figure that the $\% \mathrm{Gr}$ and $\% \mathrm{Ad}$ increase with increase in $\mathrm{kC}$ concentration up to 3.0 $w t \%$ and then decrease with further increment of polysaccharide level. The initial increase may be due to the availability of more grafting sites, where polysaccharide can be grafted. Subsequent decrease in grafting parameters, $\% \mathrm{Gr}$ and \%Ad, can be explained on the basis of increase in viscosity of the medium. This observation is in close agreement with the results obtained by Zhang and Chen ${ }^{24}$

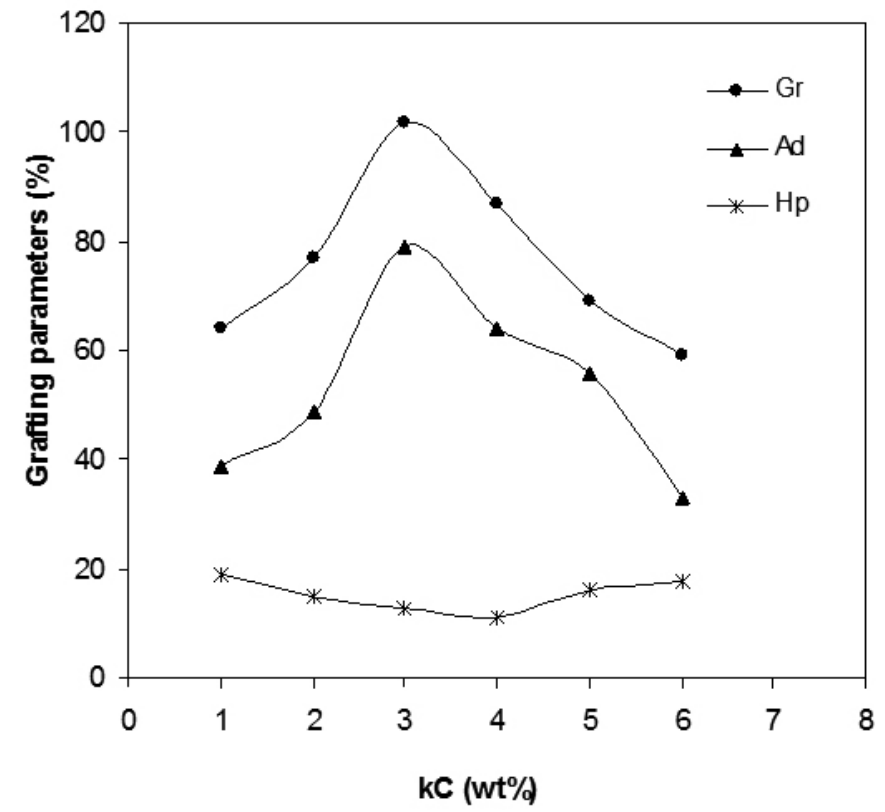

Figure 9. Grafting parameters as functions of $\mathrm{kC}$ concentration.

Reaction conditions: CAN 0.004 mol.L ${ }^{-1}$, HEMA 0.6 mol.L ${ }^{-1}$, temperature $60^{\circ} \mathrm{C}$, time $80 \mathrm{~min}$. 


\section{Effect of reaction temperature}

Figure 10 exhibits the effect of polymerization temperature on the grafting parameters. In fact, an increase in temperature upto $70^{\circ} \mathrm{C}$ increases the grafting parameters. This behavior may be related to the mobility of reactive free radical sites. Moreover, higher temperatures increase the solubility of the reactants. Temperatures higher than $70{ }^{\circ} \mathrm{C}$ disfavor the grafting parameters. At higher temperatures, the rate of termination of the growing chain is increased ${ }^{25}$ and the monomer is volatilized out to some extent ${ }^{26}$.

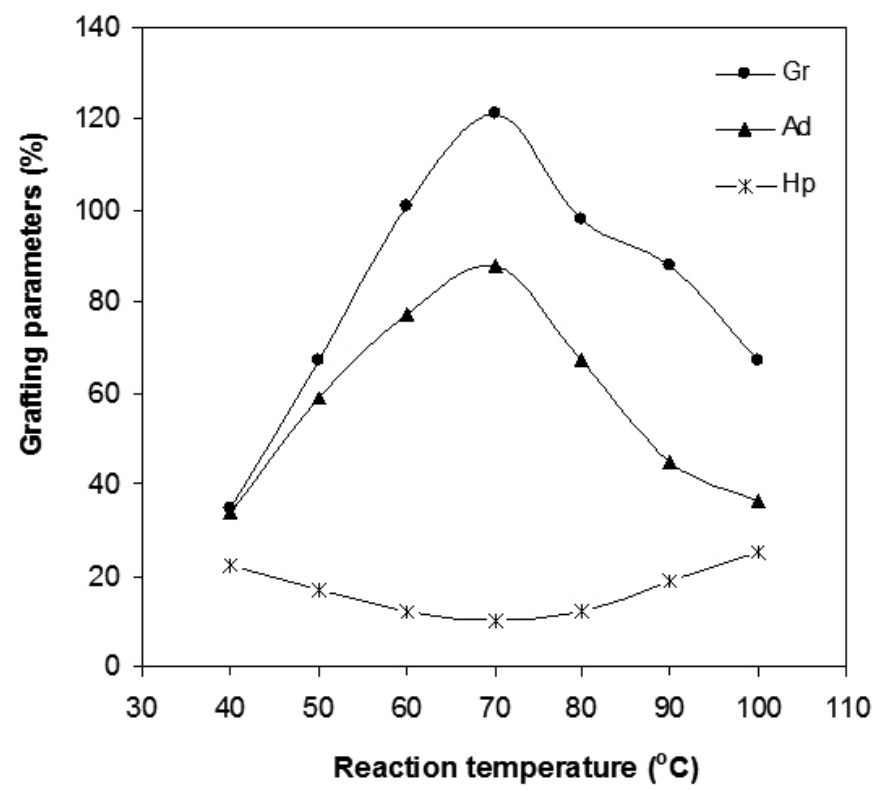

Figure 10. Effect of the reaction temperature on the grafting parameters.

Reaction conditions: $\mathrm{kC} 3 \mathrm{wt} \%$, CAN 0.004 mol.L $\mathrm{L}^{-1}$, HEMA 0.6 mol.L $\mathrm{L}^{-1}$, time $80 \mathrm{~min}$

\section{Effect of reaction time}

Grafting of HEMA onto $\mathrm{kC}$ backbones was carried out at various polymerization times as shown in Figure 11 . The $\% \mathrm{Gr}$ and $\% \mathrm{Ad}$ increased with increase in the reaction time up to $90 \mathrm{~min}$ and thereafter, these parameters gradually decreased. It is obvious that the longer the reaction time, the better the graft copolymerization yield. The grafting loss may be attributed to decrease of all the consuming reactants. In addition, the decreased number of available active free radical sites for grafting and the retardation of diffusion of reactants, because of the long grafted chains at the $\mathrm{kC}$ surface, may be other possible reasons for the diminished grafting at longer reaction times. Similar time dependency of grafting parameters was reported by others 27,28 .

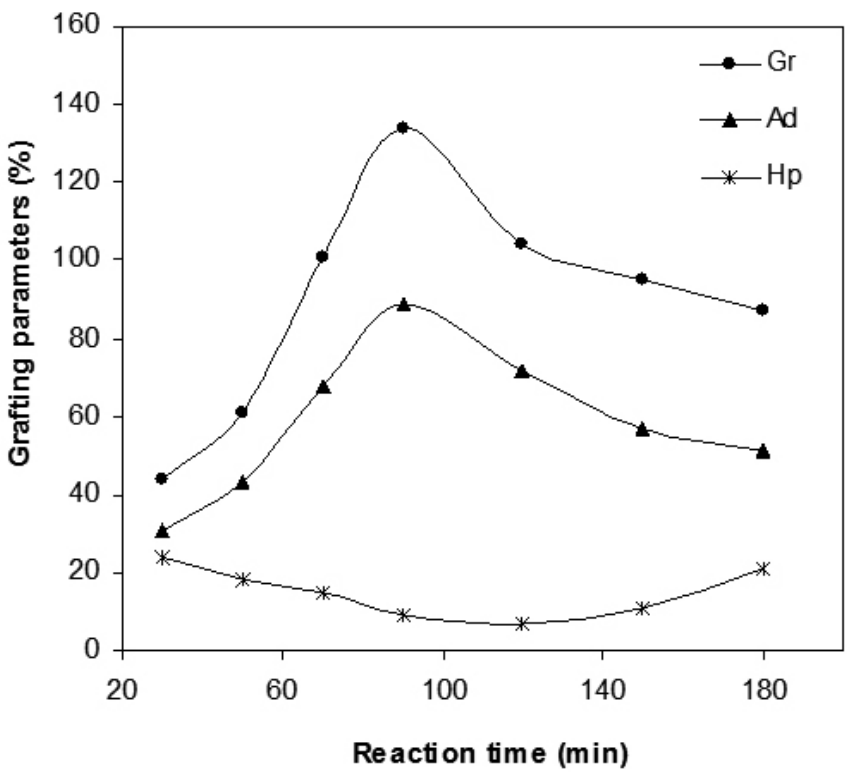

Figure 11. Effect of the reaction time on the grafting parameters.

Reaction conditions: $\mathrm{kC} 3 \mathrm{wt} \%$, CAN 0.004 mol.L $\mathrm{L}^{-1}$, HEMA 0.6 mol.L $\mathrm{L}^{-1}$, temperature $70^{\circ} \mathrm{C}$.

\section{CONCLUSIONS}

The monomer, 2-hydroxyethylmethacrylate (HEMA), can be easily graft copolymerized onto $\mathrm{kC}$ polysaccharide using $\mathrm{CAN}$ as an initiator in acidified aqueous medium. In order to prove that HEMA molecules were grafted, solubility test, FTIR spectroscopy, TGA analysis, and gravimetric analysis were used. The reaction conditions were attempted to optimize for obtaining graft copolymers with higher grafting parameters. So, the reaction conditions for achieving the maximum $\% \mathrm{Gr}(134)$ and \%Ad (89) were found to be as follows: CAN $0.004 \mathrm{~mol} / \mathrm{L}$ in 0.1 molar $\mathrm{HNO}_{3}$, HEMA $0.6 \mathrm{~mol} / \mathrm{L}, \mathrm{kC} 3 \mathrm{wt} \%$, reaction temperature $70{ }^{\circ} \mathrm{C}$, and reaction time $90 \mathrm{~min}$. Empirical polymerization rate showed a first-order dependence on the monomer concentration and a half-order dependence on the initiator concentration. According to the slope of $\mathrm{LnRg}$ versus $1 / \mathrm{T}$, the overall activation energy for graft copolymerization reaction was estimated to be $16.85 \mathrm{~kJ} / \mathrm{mol}$. As an extension of this work, the $\mathrm{kC}$ - -poly(HEMA) copolymer is being subjected to further modification to prepare thickeners and flocculants for aqueous systems.

\section{REFERENCES}

1. D.W. Jenkins, S.M. Hudson, Chem. Rev. 101, 3245 (2001).

2. G.F. Fanta, Block and Graft Copolymerization, Wiley, London, 1973.

3. A. Hebeish, J.T. Guthrie, The Chemistry and Technology of Cellulosic Copolymers, Springer-Verlag, Berlin, 1981.

4. J. Huang, W. Xu, Appl. Surf. Sci, 256, 3921 (2010).

5. S. Kalia, B.S. Kaith, J. Chil. Chem. Soc. 54, 108 (2009).

6. P.K. Pandey, A. Srivastava, J. Trpathy, K. Behari, Carbohyd. Polym. 65, 414 (2006).

7. J.M. Joshi, S.V. Kumar, Polymer, 47, 2198, (2006)

8. S.J. Metz, W.J.C. Van de Ven, J. Potreck, J. Membrance. Sci. 251, 29 (2005).

9. J.H. Ye, J.J. Dong, J.L. Lu, X.Q. Zheng, J. Jin, H. Chen, Y.R. Liang, Carbohyd. Polym. 81, 441 (2010).

10. I. Silva, M. Gurruchaga, I. Goñi, Carbohyd. Polym. 76, 593 (2009).

11. B. Singh, N. Chauhan, S. Kumar, Carbohyd. Polym. 73, 446 (2008).

12. G. Mino, S. Kaizerman, J. Polym. Sci. 31, 242 (1958).

13. G. Fu, J. Zhao, H. Yu, L. Liu, B. He, React. Funct. Polym. 67, 442 (2007).

14. P. Lv, Y. Bin, Y. Li, R. Chen, X. Wang, B. Zhao, Polymer, 50, 5675 (2009).

15. A. Pourjavadi, M.J. Zohuriaan-Mehr, A. Pooraghaberar, H. Hosseinzadeh, J. Polym. Mater. 21, 351, (2004). 
16. R.E. Kirk, D.F. Othmer, Encyclopedia of Chemical Technology, 4rd edition, John Wiley \& Sons, New York, 1992.

17. B.N. Mishra, J. Kishore, M. Kanthwal, I.K. Mehta, J. Polym. Sci. Part A: Polym. Chem, 24, 2209 (1986).

18. A. Pourjavadi, M.J. Zohuriaan-Mehr, Starch/Starke, 54, 140 (2002).

19. M.J. Zohuriaan-Mehr, A. Pourjavadi, M. Sadeghi, Iran. Polym. J. 14, 131 (2005).

20. N. Isiklan, F. Kursun, M. Inal, Carbohyd. Polym., 79, 665 (2010).

21. F. Carrillo, B. Defays, X. Colom, Europ. Polym. J. 44, 4020 (2008).
22. K.C. Gupta, S. Sahoo, K. Khandekar, Biomacromolecules, 3, 1087 (2002).

23. L. Rahman, S. Silong, W.M. Zin, M.Z. Ab Rahman, M. Ahmad, J. Haron, J. Appl. Polym. Sci. 76, 516 (2000).

24. L.M. Zhang, L.Q. Chen, J. Appl. Polym. Sci. 83, 2755 (2002).

25. T. Sun, P. Xu, Q. Liu, J. Xue, W. Xie, Europ. Polym. J. 39, 189 (2003).

26. K. Behari, P.K. Pandey, R. Kumar, K. Taunk, Carbohydr. Polym. 46, 185 (2001).

27. R. Ojah, S. K. Dolui, Biores. Tech. 97, 1529 (2006).

28. K.C. Gupta, S. Sahoo, Biomaterials, 2, 239 (2001). 